\title{
Verletzungen des Beckens
}

\author{
Axel Gänsslen, Annelie-Martina Weinberg
}

\section{Zusammenfassung}

Kindliche Beckenverletzungen sind selten und werden auch heute noch nicht selten übersehen. Eine klar strukturierte Primärdiagnostik muss deshalb obligat durchgeführt werden, da auch instabile Verletzungen des kindlichen Beckenrings oftmals nur schwer zu diagnostizieren sind. Das Primär- und Definitivmanagement dieser Verletzungen orientiert sich im Wesentlichen an dem des Erwachsenen. Aufgrund der potenziellen negativen Langzeitfolgen sollte die Therapie der instabilen Beckenringverletzungen osteosynthetisch mittels kindadaptierter Stabilisierungstechniken erfolgen. Trotzdem muss mit Wachstumsstörungen gerechnet werden. Entsprechend sind regelmäßige klinisch-radiologische Kontrollen bis zum Abschluss des Wachstums zu fordern. Verletzungen der kindlichen Wachstumsfuge des Azetabulums sind schon aufgrund der schwierigen radiologischen Beurteilung selten. Entsprechend sind Angaben zur Inzidenz in der Literatur nicht einheitlich. Schwierigkeiten bestehen zusätzlich darin, bis zu welchem Alter das Azetabulum als „kindlich“ angesehen werden kann. Für die kindlichen Verletzungen hat sich die Klassifikation nach Salter-Harris bewährt. Die Mehrzahl dieser Verletzungen kann konservativ erfolgreich therapiert werden. Bei starker Dislokation oder intraartikulären Pathologien kommen auch offene Verfahren zur Anwendung. Aufgrund der Hauptgefahr der Ausbildung einer posttraumatischen Gelenkdysplasie sollten regelmäßige Kontrolluntersuchungen bis zum Wachstumsabschluss durchgeführt werden. Die Langzeitergebnisse können insgesamt als zufriedenstellend gewertet werden.

\section{Pelvic Injuries in Children}

Pelvic injuries in children are rare and are overlooked frequently even today. An adequately structured primary diagnosis must therefore be mandatory. The primary management of these injuries is geared to that of adults. Due to the potential negative long-term consequences, the treatment of unstable pelvic ring injuries should be per- formed by child-adapted stabilisation techniques. Nevertheless, growth disturbances occur in rare cases. Therefore, frequent clinical and radiological controls are proposed until the completion of growth. Injuries to the acetabular growth plate are rarely seen, especially due to the difficult radiological assessment. Accordingly, the incidence in the literature is not uniform. Additionally, it is difficult to decide at which age the acetabulum is considered to be that of a child. The classification according to Salter-Harris in smaller children and the Letournel classification in adolescents are the most frequently used ones. The majority of acetabular injuries can be treated conservatively. Displaced fractures and the presence of intra-articular pathologies are indications for open reduction and internal fixation. The main longterm sequelae are the potential formation of a post-traumatic joint dysplasia which should be checked until the completion of growth. The long-term results can be considered satisfactory in the majority of cases.

\section{Einleitung}

Das kindliche Beckenskelett weist im Unterschied zum Erwachsenen wesentliche Unterschiede auf. Der kindliche Knochen ist verformbarer und absorbiert durch den höheren Knorpelanteil mehr Energie, bevor er bricht $[3,9,10]$.

Energie kann auch nach Entstehung einer Fraktur weiter aufgenommen werden. Die erhöhte Elastizität vermindert dabei den Schutz der intrapelvinen Or-

OP-JOURNAL 2010; 26: 116-122

(c) Georg Thieme Verlag KG Stuttgart · New York DOI http://dx.doi.org/10.1055/s-0030-1250171 gane. Entsprechend kann es trotz fehlender Frakturzeichen zu Verletzungen der inneren Organe kommen.

Die einwirkende Kraft ist deshalb bei Vorliegen von Frakturen oder Gelenkverletzungen als deutlich größer anzusehen als beim Erwachsen. Beim Vorliegen auch einfacher Frakturen muss deshalb immer mit inneren Verletzungen gerechnet werden. So treten bei der kindlichen Beckenfraktur doppelt so häufig pelvine Begleitverletzungen auf als beim Erwachsenen.

Eine isolierte Schambeinastfraktur kann beim Kleinkind also schon zu erheblichen inneren Verletzungen geführt ha- ben. Beim Auftreten mehrerer Frakturen im kindlichen Beckenring muss deswegen nahezu immer mit intraabdominellen und pelvinen Begleitverletzungen gerechnet werden.

Bezüglich des Langzeitergebnisses konnte die früher weitverbreitete Ansicht, dass alle Beckenverletzungen im Kindesalter aufgrund der wachstumsbedingten Kompensationsmechanismen folgenlos ausheilen, zwischenzeitlich in mehreren Untersuchungen widerlegt werden $[4,8]$. 

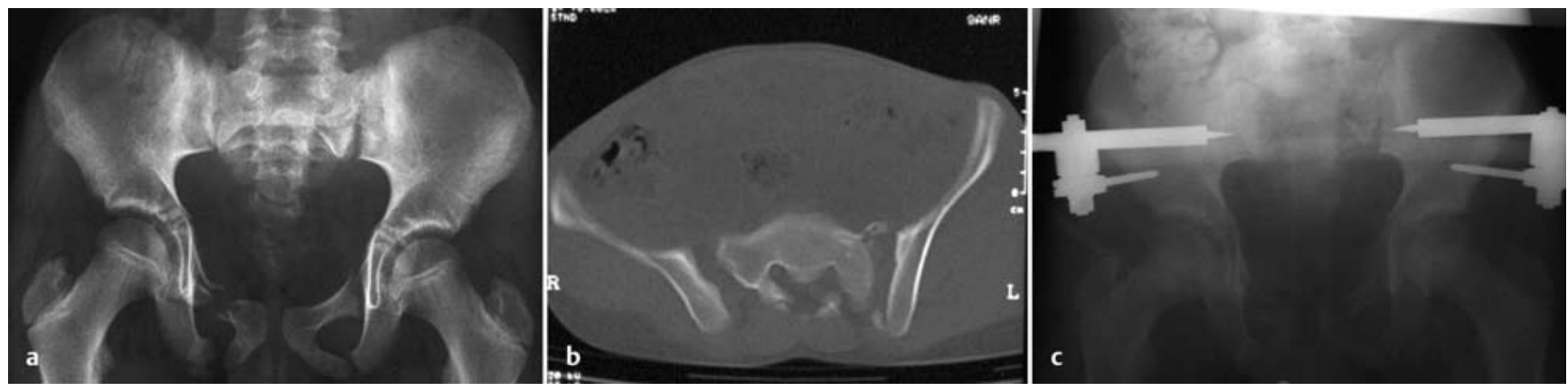

Abb. 1 a bis c Elfjähriger Junge nach PKW-Unfall mit beidseits instabilem vorderen und hinteren Beckenring. Notfallstabilisierung mittels Beckenzwinge und pelviner Tamponade und sekundäre Stabilisierung des vorderen Beckenrings mittels supraazetabulärem Fixateur externe.

\section{Beckenring}

Der kindliche Beckenbruch ist auf die gleichen äußeren Kräfte zurückzuführen wie beim Erwachsenen [9]. Das Frakturmuster weist jedoch erhebliche Unterschiede auf.

Während es beim Erwachsenen nach lateraler Kompression in der Regel zur Instabilität einer Beckenhälfte kommt, führt der gleiche Mechanismus beim Kind eher zur Fraktur des Azetabulums. Die Kraft wirkt über den Schenkelhals auf das Azetabulum ein, sodass abhängig von der Hüftposition vergleichbare Frakturtypen wie beim Erwachsenen entstehen. Anteroposteriore Kompression und axiale Translationen bewirken in beiden Altersgruppen ähnliche Frakturformen.

Die klinische Untersuchung entspricht im Wesentlichen dem Ablauf beim Erwachsenen. Neben der Inspektion der Beckenregion sollte v.a. die klinische Beckenstabilität beurteilt werden, eine Untersuchung der Orificii ist obligat. Begleitverletzungen sind häufig an den unteren Extremitäten, insbesondere am Femur zu erwarten [9].

\section{Radiologische Diagnostik}

Hauptproblem der ausschließlich konventionell radiologischen Diagnostik bleibt eine nicht geringe Rate übersehener Verletzungen, v.a. auch im Bereich des dorsalen Beckenrings.

Neben der obligaten Beckenübersichtsaufnahme empfehlen wir insbesondere beim mehrfach verletzten Kind deshalb zwingend die Durchführung einer CTUntersuchung mit multiplanaren Reformationen.

Ergänzend kann auch eine MRT-Untersuchung im Verlauf bei nicht erklärbaren Beschwerden indiziert sein.

\section{Klassifikation}

Im deutschsprachigen Raum hat sich die AO- bzw. OTA-Klassifikation des Erwachsenen auch für kindliche Beckenringverletzungen durchgesetzt.

\section{Primärbehandlung}

Aufgrund des hohen Risikos begleitender intra- und extrapelviner Verletzungen beim Vorliegen von Frakturen werden kindliche Beckenfrakturen bis zum Beweis des Gegenteils zunächst als lebensbedrohliche Verletzungen angesehen $[8,9]$.

Die Primärbehandlung orientiert sich entsprechend primär am ATLS-Konzept und dem jeweiligen klinikinternen Polytraumaalgorithmus und ist damit vergleichbar der Behandlung beim Erwachsenen.

Speziell für das Becken sind die klinische Stabilitätsprüfung, die Analyse der Körperöffnungen, das Vorliegen von ausgedehnten Weichteilverletzungen (offene Frakturen, Morell-Levallé-Läsionen nach Überrolltrauma oder Scherverletzungen auch im Bereich der Lumbalregion und der Oberschenkelregion), die begleitende Sonografie von Abdomen und Becken (FAST) sowie eine adäquate radiologische Diagnostik für die Einschätzung der Schwere der Beckenverletzung maßgebend.

Daneben muss natürlich auf lebensgefährdende extrapelvine Begleitverletzungen geachtet werden.

Die Art der Primärtherapie orientiert sich am Becken an der mechanischen Instabilität des Beckens, der Kreislaufinstabilität (Hämoglobinwert $<8 \mathrm{~g} / \mathrm{dl}$, Base Exzess $<-6 \mathrm{mmol} / \mathrm{l}$ ) und der lokalen Begleitverletzungen (s.o.).
Die hohe Kompensationsfähigkeit des kindlichen Organismus hinsichtlich eines traumatisch-hämorrhagischen Schocks muss dabei besonders beachtet werden.

Die wesentliche primäre Behandlungsmaßnahme ist die mechanische Stabilisierung des Beckenrings.

Dies erfolgt im Regelfall mittels supraazetabulärem Fixateur externe (Abb. 1). Als Alternativen kommen die Tuchumschlingung oder die Anwendung eines Beckengürtels infrage. Beim adoleszenten Kind ist auch die Anwendung der Beckenzwinge in Betracht zu ziehen (Abb. 1).

Zur primären Therapie einer durch die Beckenverletzung bedingten Kreislaufinsuffizienz sehen wir die extraperitoneale pelvine Tamponade als Maßnahme der ersten Wahl, da aufgrund des relativen Zeitmangels bei fehlender hämodynamischer Kompensationsmöglichkeit eine mögliche Angiografie und Embolisation einen zu großen Zeitverlust bedeuten würde. Die operative Technik entspricht der des Erwachsenen [8,9].

Insbesondere bei den nicht seltenen kindlichen Überrollverletzungen (Abb. 2) kann es zu ausgedehnten geschlossenen, aber auch offenen Décollement-Verletzungen (Morell-Lavallé-Läsion) kommen, die regelhaft mehrfache Revisionsoperationen mit plastischer Deckung nach sich ziehen. Es entstehen große Seromhöhlen, die auch bei geschlossenen Weichteilverhältnissen kontaminiert sein können. Durch die Weichteilspannung entstehen ausgedehnte Nekrosen.

Gegenwärtig besteht noch Uneinigkeit, ob eine Drainage über kleine Inzisionen oder die weite Eröffnung mit Débridement und offener Wundbehandlung durchgeführt werden soll. Im eigenen 
Vorgehen hat sich Letzteres als Behandlungsmethode durchgesetzt.

\section{Definitivtherapie}

Die wachstumsbedingte Kompensationsfähigkeit und das Ausmaß des Remodellings von Fehlstellungen am kindlichen Beckenring sind v.a. bei den translationsinstabilen Verletzungen reduziert. Eine folgenlose Ausheilung wird nicht regelhaft erzielt.

Trotzdem ist aufgrund der o.g. anatomischen Besonderheiten, v. a. der höheren Elastizität von Knochen und Bandverbindungen, die nicht operative Therapie wesentlich häufiger indiziert und auch suffizient möglich als beim Erwachsenen.

\section{Nicht operative Therapie}

In den meisten Fällen liegt ein allenfalls nur geringer Stabilitätsverlust des kindlichen Beckenrings vor, sodass bei entsprechend geringer Frakturfehlstellung die nicht operative Therapie zu einer komplett oder nahezu anatomischen Ausheilung führt (Abb. 3).

Entsprechend werden die meisten Beckenrandfrakturen, Apophysenabrisse, Beckenringverletzungen mit isolierten Schambeinastfrakturen und gesicherter fehlender dorsaler Verletzung, in Abhängigkeit von vorliegenden Begleitverletzungen, konservativ-funktionell behandelt $[1,9,11]$.

Nach kurzfristiger Bettruhe und Analgesie erfolgt die schmerzadaptierte Mobilisation ggf. an Unterarmgehstützen.

Klinische und radiologische Verlaufskontrollen erfolgen nach 6 Wochen und zur Sicherung eines normalen Wachstums nach 1 Jahr. Bei unklaren Befunden im Sinne von potenziellen Wachstumsstörungen erfolgen jährliche Verlaufskontrollen bis Wachstumsende.

\section{Operative Therapie}

Nur die wenigsten kindlichen Beckenverletzungen müssen operativ stabilisiert werden.

Wesentliche Indikationen zur operativen Therapie sind:

- stark dislozierte Verletzungen bei TypA-Verletzung mit Gefahr von Organverletzungen (z.B. drohende Blasenperforation bei verdrehtem Scham-

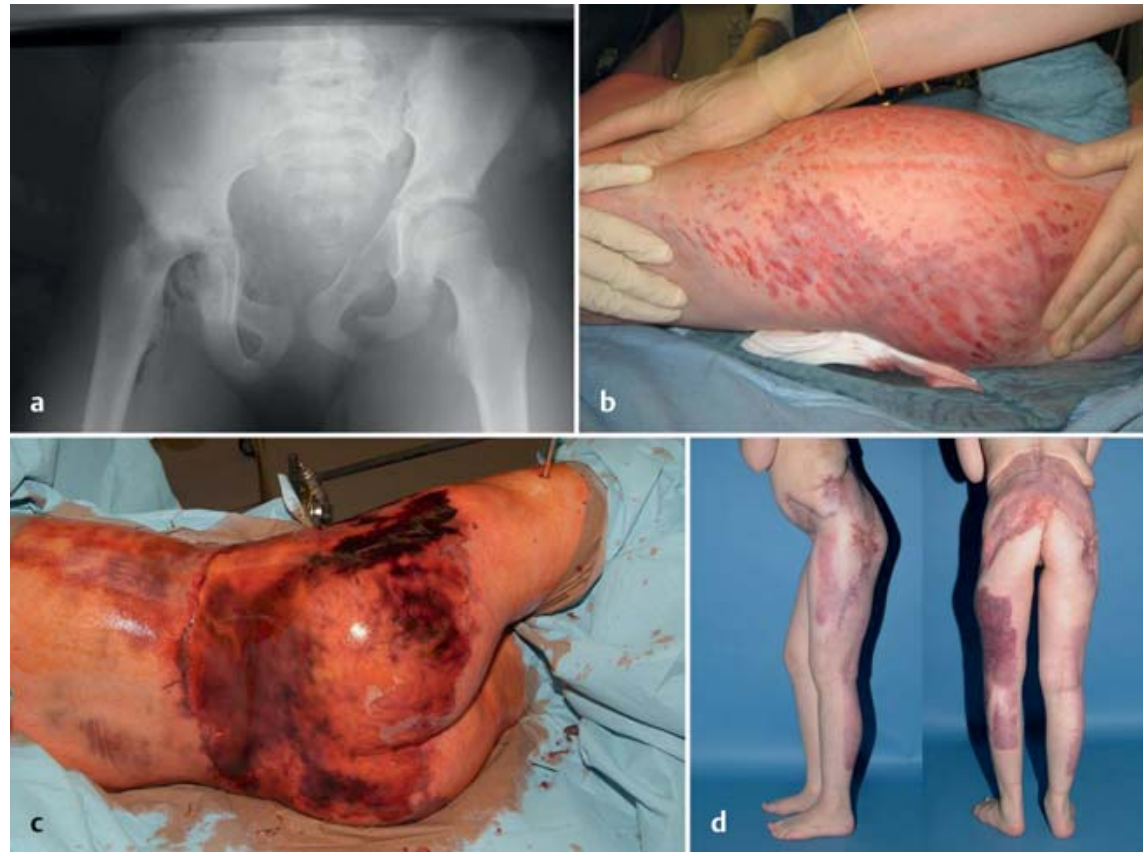

Abb. $\mathbf{2}$ a bis $\mathbf{d}$ Zehnjähriges Mädchen nach Überrolltrauma mit beidseitigen Schambeinastfrakturen und transiliakaler Luxationsfraktur links mit ausgedehnter Décollementverletzung im gesamten Beckenbereich. Nach offenem Débridement und sekundärer plastischer Deckung bestehen deutliche Funktionseinschränkungen.
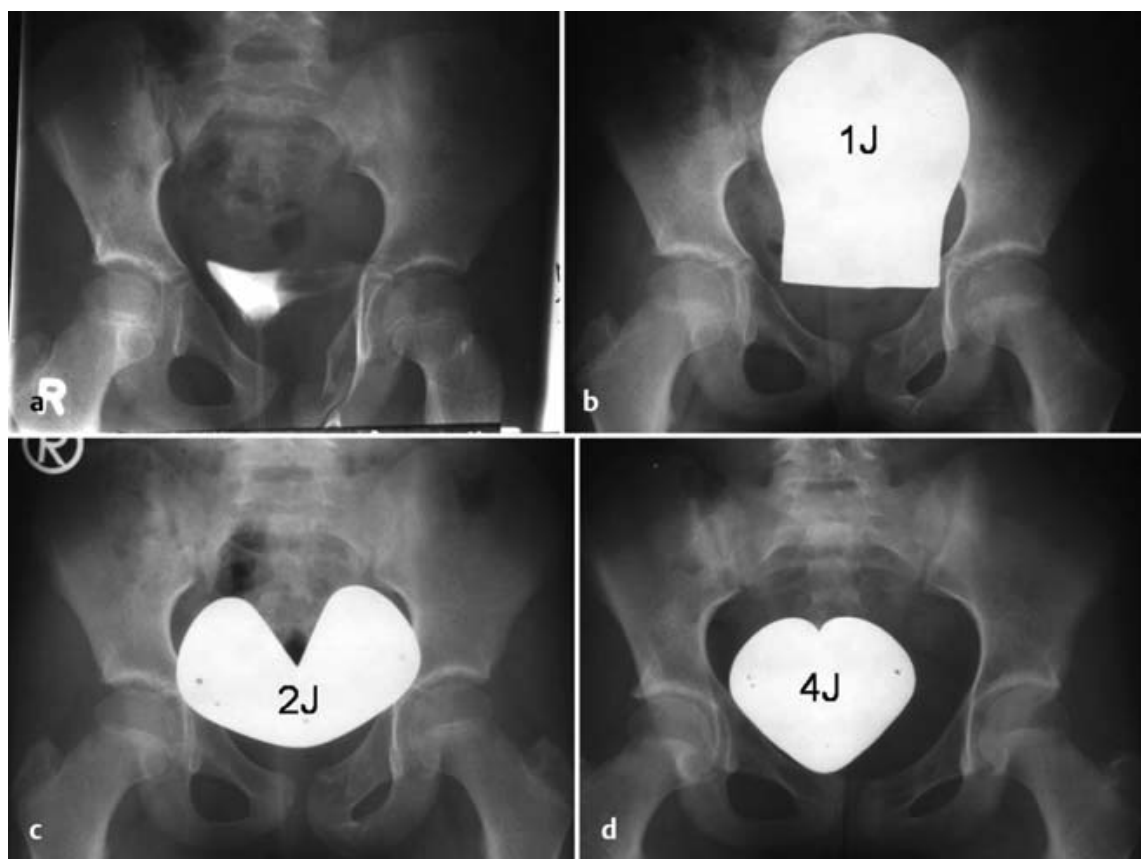

Abb. 3 a bis d Achtjähriges Mädchen nach seitlichem Anpralltrauma als Fußgängerin mit pubischen Frakturen links und Sakrumfraktur rechts. Radiologische Verlaufskontrollen nach 1, 2 und 4 Jahren mit nahezu anatomischem Remodelling.

beinastfragment, drohende Hautperforation bei Beckenrandfrakturen)

- stark dislozierte Typ-B- und -C-Verletzungen

- klinisch instabile Beckenringverletzungen vom Typ B und C 

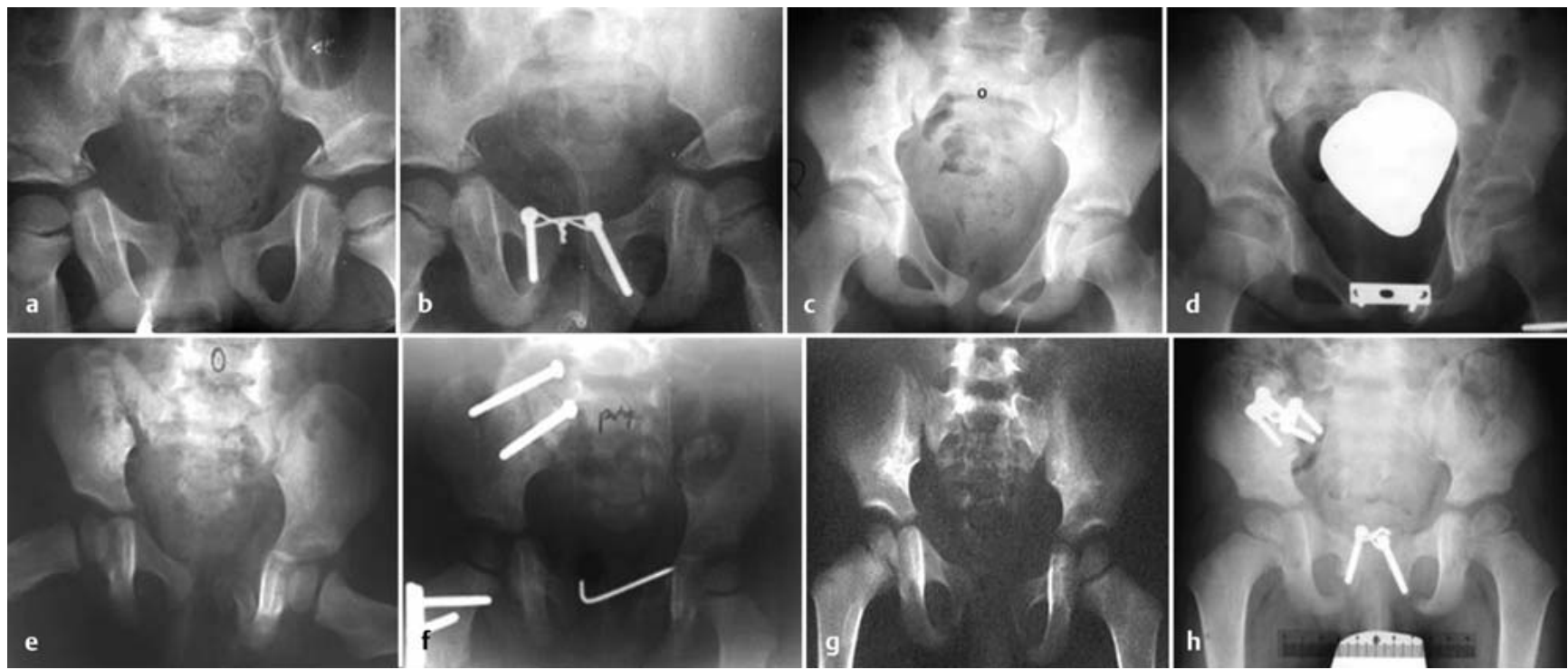

Abb. $\mathbf{4}$ a bis $\mathbf{h}$ Möglichkeiten kindadaptierter Osteosynthesetechniken am vorderen und hinteren Beckenring. Im oberen Bildabschnitt sind mögliche Techniken zur Symphysenstabilisierung mit Schrauben-/Cerclagen-Stabilisierung und Kleinfragment-Plattenosteosynthese gezeigt. Im unteren Abschnitt werden eine lliumerschraubung mit transpubischer Spickdrahtosteosynthese sowie eine modifizierte ventrale Plattenosteosynthese des SI-Gelenks gezeigt.

Verschiedene Techniken sind in der Literatur berichtet worden (dorsale interne Osteosynthesen, perkutane KirschnerDraht-Fixationen von SI-Gelenk und anderen Verletzungen des hinteren Beckenrings).

Als Standardverfahren kann die Stabilisierung mittels Fixateur externe angesehen werden.

Daneben wurden im eigenen Vorgehen altersabhängig Osteosynthesetechniken eingesetzt [9].

Bei Kindern im Alter von 12-14 Jahren können den Erwachsenen vergleichbare Osteosynthesetechniken eingesetzt werden. Hier ist jedoch besonders auf die Überbrückung der artikulären Beckenverbindungen zu achten bzw. die Kreuzung von Wachstumsbereichen im Os sacrum bei Verschraubungen. In Abhängigkeit von der Größe des Beckens müssen die Implantate an die lokale Anatomie angepasst werden (z.B. Kleinfragmentplatten zur Symphysen- und SI-Gelenkstabilisierung, $4,5 \mathrm{~mm}$ transiliosakrale Schrauben).

Unterhalb dieser Altersgruppe sind die Implantate des Erwachsenen zu groß. Entsprechend kommen Modifikationen zur Anwendung (Abb. 4):

- Symphysensprengung: Schrauben-/ Cerclagen-Osteosynthese, transossäre Nahttechniken, Plattenosteosynthese mit Drittelrohrplatte oder Kleinfragmentplatte

- transpubische Instabilität: supraazetabulärer Fixateur externe, ggf. offene Reposition und Stabilisierung mittels Spickdraht/Kleinfragmentschraube

- Beckenrandfraktur: offene Reposition und Spickdraht- oder Schraubenosteosynthese

- SI-Sprengung: ventrale Plattenosteosynthese mit $\mathrm{H}$-Platte, transiliosakrale Kleinfragmentverschraubung

- Sakrumfrakturen: perkutane transiliosakrale Kirschner-Draht- oder Kleinfragmentverschraubung

Die Nachbehandlung erfolgt nach instabilen Verletzungen mit Teilbelastung der betroffenen Seite (dorsaler Beckenring) für 4-8 Wochen. Eine Implantatentfernung wird nach 3-6 Monaten durchgeführt.

\section{Komplikationen}

Komplikationen im Bereich des knöchernen und ligamentären Beckenrings sind bedingt durch eine Überschreitung der physiologischen Korrekturpotenz (Abb.5). Als Langzeitfolgen sind beschrieben $[8,13]$ :

- Fehlstellungen

- hypoplastische Beckenhälften

- Ankylosen von Symphyse oder SI-Gelenk

- posttraumatische Hüftdysplasien (s.u.)

- Pseudarthrosen

- Beinlängendifferenzen
Zusätzlich können Komplikationen durch Folgen der pelvinen Begleitverletzungen bedingt sein. Hier sind urogenitale und neurologische Folgeschäden zu nennen, die durchaus relevanten Einfluss auf das Langzeitergebnis haben.

\section{Langzeitergebnisse}

Ergebnisse nach kindlichen Beckenringverletzungen sind in der Literatur zwar mittlerweile häufig publiziert [12]. Die Einschätzung ist jedoch schwierig, da insbesondere das Einschlusskriterium Patientenalter, die verwendeten Klassifikationen und die Outcome-Beurteilung deutlich differieren und meistens keine konsekutiven Serien analysiert wurden.

Insgesamt kann davon ausgegangen werden, dass gerade bei Kindern nach stabilen Typ-A- und -B-Verletzungen nach konservativ-funktioneller Therapie in $>90 \%$ gute radiologische und funktionelle Ergebnisse zu erzielen sind. Auch heilen die meisten Typ-C-Verletzungen folgenlos aus.

Es ist gegenwärtig noch nicht klar, welche Typ-C-Verletzungen von einer operativen Therapie profitieren, obwohl erste Langzeitergebnisse nach operativer Therapie gute Ergebnisse zeigen.

Einigkeit besteht darin, dass nach komplexen Beckenverletzungen (zusätzlicher peripelviner Weichteilschaden) schlechtere Ergebnisse resultieren mit häufig 
langfristigen urogenitalen und neurologischen Folgeschäden.

Typ-C-Verletzungen sind allerdings die Verletzungen, die am häufigsten mit Wachstumsschäden assoziiert sind, wobei eine funktionelle Konsequenz nicht immer vorliegen muss.

\section{Azetabulumfrakturen}

Verletzungen der kindlichen Wachstumsfuge des Azetabulums sind schon aufgrund der schwierigen radiologischen Beurteilung selten. Entsprechend sind Angaben zur Inzidenz in der Literatur nicht einheitlich. Schwierigkeiten bestehen zusätzlich darin, bis zu welchem Alter das Azetabulum als „kindlich“ angesehen werden kann.

Kindliche Verletzungen des Azetabulums sind selten, insbesondere bei Beteiligung der azetabulären Wachstumsfuge. Die Häufigkeit kindlicher Azetabulumfrakturen liegt bei etwa $0,3 \%$. Innerhalb dieser Gruppe wird die Inzidenz der Verletzungen mit Wachstumsfugenbeteiligung mit 4-20\% angegeben.

Für das Entstehen einer kindlichen Azetabulumfraktur wird regelhaft ein Hochrasanztrauma, v.a. nach Verkehrsunfällen, verantwortlich gemacht. Eine Schädigung der Wachstumsfuge wird dabei direkt durch das Trauma verursacht.

\section{Röntgendiagnostik}

Die radiologische Diagnose einer kindlichen Azetabulumverletzung ist häufig schwierig.

Es ist immer die Durchführung einer Beckenübersichtsaufnahme zu fordern. Schrägaufnahmen des Azetabulums (Ala-, Obturatoraufnahme) können hilfreich sein, ggf. ist bei Bedarf die Durchführung einer Computertomografie erforderlich und sollte bei klinischem Verdacht durchgeführt werden. Das CT dient dabei in erster Linie dem Nachweis osteochondraler Fragmente, die auf den konventionellen Aufnahmen nicht zu sehen sind. Frakturen des kindlichen Azetabulums imponieren konventionell-radiologisch als (Abb. 6):

- Dislokationen im Wachstumsfugenbereich

- Unterbrechung der Linea iliopectinea

- Zeichen eines intraartikulären Ergusses mit abgespanntem Kapselschatten

- Asymmetrie der Tränenfigur im Seitenvergleich

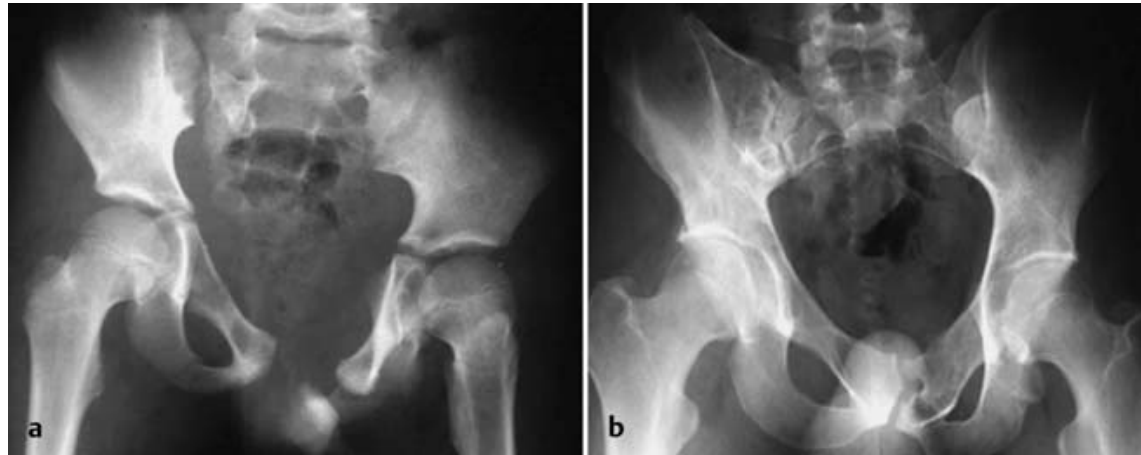

Abb.5a und b Hypoplasie der linken Beckenhälfte und Arthrose des SI-Gelenks rechts nach Überrolltrauma mit Typ-C-Verletzung rechts und Typ-B-Verletzung links.
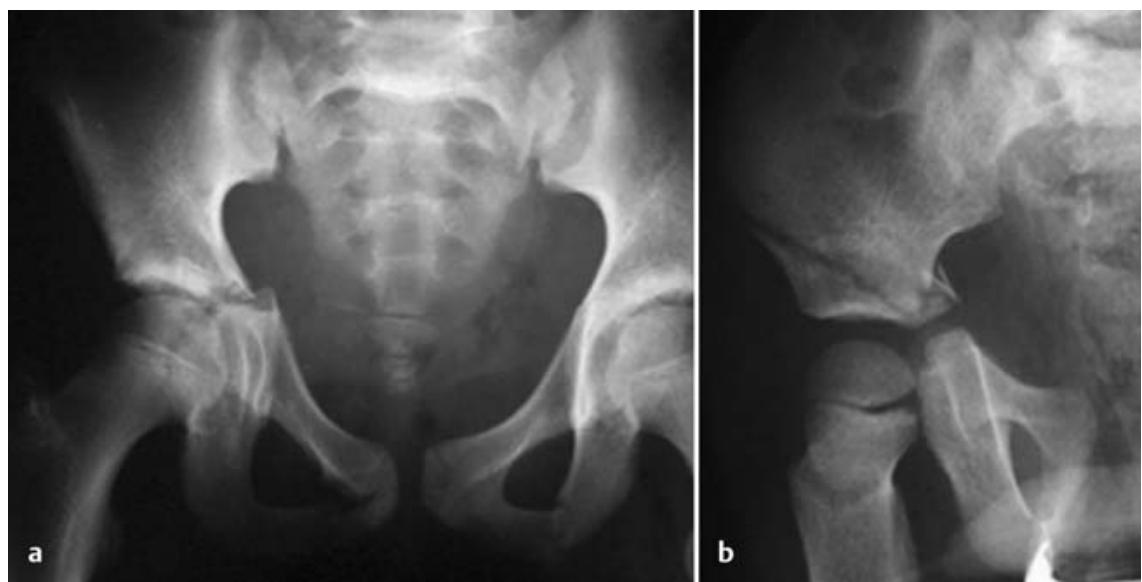

Abb.6a und b Typische Morphologie von Azetabulumverletzungen im Kleinkindalter. Epiphysiolyse Typ Salter I (rechts) und Epiphysiolyse mit metaphysärer Verletzung Typ Salter II (links).

Der Wert der Sonografie zum Nachweis eines intraartikulären Ergusses ist nicht bekannt, ebenso die Wertigkeit einer MRT-Diagnostik.

Typischerweise liegt bei kindlichen Frakturen des Azetabulums eine Epiphysiolyse mit und ohne metaphysäre Beteiligung vor. Häufig besteht eine zusätzliche Verletzung des Beckenrings [2].

Ein sich entwickelndes Os acetabuli kann zu Schwierigkeiten in der Differenzierung zwischen Fraktur und normalem Wachstumsverhalten führen.

Aufgrund der schwierigen Diagnostik und der damit verbundenen prognostisch ggf. ungünstigen Gefahr des Übersehens der Verletzung sollte eine radiologische Verlaufskontrolle (nach 2-4 Wochen) durchgeführt werden. Sekundär nachweisbare Periostreaktionen können dabei Hinweise auf eine azetabuläre Verletzung geben.

\section{Klassifikation}

Die Klassifikation ist nur selten anhand der beim Erwachsenen angewandten Letournel-Klassifikation möglich. Viele Azetabulumfrakturen bei älteren Kindern lassen sich nach Letournel klassifizieren. Die übrigen werden nach der Einteilung von Bucholz klassifiziert.

Bucholz unterscheidet 2 wesentliche Verletzungstypen [2]:

- Verletzungen der Y-Fuge ohne/mit metaphysärer Beteiligung (Abb.6): entstehen durch Scherkräfte mit Krafteinwirkung gegen das Os ischium oder Os pubis oder das proximale Femur. Diese Verletzungen entsprechen den Salter-Harris-Typ-I- und -II-Verletzungen mit guter Prognose und meist normalem Wachstumsverhalten.

- Kompressionsverletzung der Wachstumsfuge (Salter-Harris Typ V) mit schlechterer Prognose.

Andere Einteilungen unterscheiden stabile, nicht dislozierte Frakturen (1), instabile, dislozierte Frakturen (2) und 


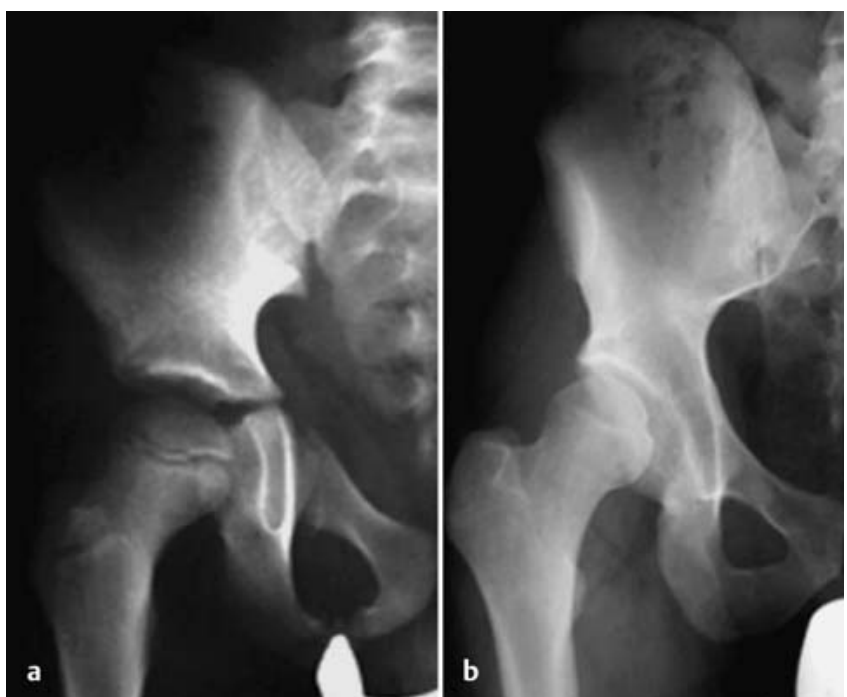

Abb. 7 a und $b$ Komplikation nach Salter--I-Verletzung des rechten Azetabulums. Kontrolle nach 18 Jahren mit Ausbildung einer posttraumatischen Dysplasie.
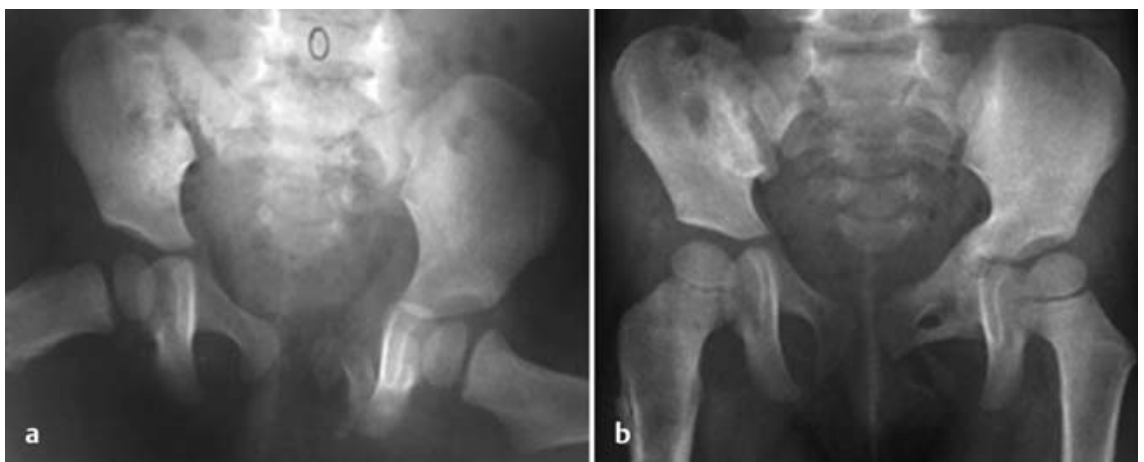

Abb. $\mathbf{8}$ a und $\mathbf{b}$ Komplikation nach Salter-I-Verletzung des linken Azetabulums. Nach 1,5 Jahren Epiphysiodese des vorderen Pfeilers des Azetabulums.

zentrale Luxationsfrakturen des Hüftgelenks (3) bzw. Pfannendachfrakturen (1), Pfannengrundfrakturen (2), Pfannenrandfrakturen (3) oder zentrale Luxationsfrakturen des Hüftgelenks (4).

\section{Therapie}

In der Behandlung der Azetabulumfraktur des Kindes dominiert die konservative Therapie $[9,11]$. Sie umfasst Bettruhe, Lagerung und/oder Extensionsbehandlung [7].

Stabile, nicht dislozierte Frakturen werden von einigen Autoren mittels Bettruhe, zeitnaher nicht gelenkbelastender Krankengymnastik und Teilbelastung an Stützen nach 1 Woche behandelt. Instabile, dislozierte Frakturen wurden mit Extension für 6 Wochen und daran anschließend weiteren 6 Wochen Gehen mit Stützen ohne Belastung therapiert.

Andere Autoren favorisieren eine suprakondyläre Steinmann-Nagel-Extension für 3-4 Wochen mit anschließender
Bettruhe für 2-4 Wochen oder die Anlage eines Beckengipses für 3-4 Wochen.

Im eigenen Vorgehen hat sich die frühfunktionelle Behandlung mit schmerzadaptierter Mobilisation bei undislozierten Frakturen durchgesetzt. Bei dislozierten Frakturen erfolgt altersabhängig eine 4-6-wöchige Entlastung mit anschließendem Belastungsaufbau.

Die offene Reposition und interne Fixation mit dem Ziel einer anatomischen Reposition und Retention ist bei dislozierten und instabilen Frakturen indiziert $[5,6,9]$.

Indikationen zur operativen Therapie sind:

- persistierende Interponate

- Luxationstendenz durch fehlende Gelenkkongruenz

- persistierende Fragmentdislokation

Als Fixationsmöglichkeiten werden Einzelschraubenosteosynthesen, KirschnerDraht-Osteosynthesen, transossäre Näh- te und Plattenstabilisierungen angegeben.

Hinsichtlich des postoperativen Belastungsaufbaus kann derzeit keine allgemeingültige Empfehlung gegeben werden.

Es wird von 4-8 Wochen Hüftgelenksentlastung ohne oder mit Extension, beschwerdeabhängiger Mobilisation und Teilbelastung für 6-12 Wochen und anschließender Vollbelastung berichtet.

Ziel der operativen Behandlung muss aber auf jeden Fall die sparsame Osteosynthese sein, die das Pfannenwachstum nicht behindert.

\section{Komplikationen}

Ähnlich wie für Erwachsene sind verschiedene Spätkomplikationen auch nach kindlichen Azetabulumfrakturen angegeben:

- Entwicklung einer posttraumatischen Dysplasie des Hüftgelenks (Abb. 7)

- Entwicklung einer posttraumatischen Hüftkopfnekrose

- Beinlängendifferenzen

- posttraumatische Arthrose

- Epiphysiodesen (Abb. 8)

Insgesamt finden sich allerdings nur wenige Analysen hinsichtlich Langzeitfolgen kindlicher Azetabulumfrakturen.

Die Entwicklung einer posttraumatischen Azetabulumdysplasie ist dabei am besten untersucht.

Ursächlich für die Ausbildung einer Dysplasie können periartikuläre Verknöcherungen von abgesprengten Knorpelstückchen sein, eine Vernarbung der Y-Fuge oder die Zerstörung der Germinalzellschicht, Gefäßverletzungen des Knorpels, knöcherne Überbrückung des Azetabulumknorpels durch Ossifikation eines Hämatoms oder fugenkreuzende Kallusbrücken.

Wann und in welchem Ausmaß sich eine Dysplasie entwickelt, ist nicht bekannt. In tierexperimentellen Untersuchungen wurden Anteile der Y-Fuge fusioniert mit der Folge einer altersabhängigen Entwicklung einer Dysplasie.

Die isolierte Fusion zwischen Os ilium und Os pubis führte nur $\mathrm{zu}$ geringen Wachstumsstörungen, was den klinischen Beobachtungen entsprach. 
Als radiologische Kennzeichen der Dysplasie sind Beckenasymmetrie, Coxa parva, laterale Subluxation des Femurkopfs, Verbreiterung der Tränenfigur, Gelenkinkongruenz und verkleinerter CenterEdge-Winkel beschrieben. Zusätzlich kann es zu einer Abflachung des Azetabulums, Verdickung der medialen Wand und Subluxation des Hüftkopfs, Abflachung des Azetabulums, verdicktem Pfannenboden, schlechter Überdachung des Hüftkopfs, Verdickung der medialen Azetabulumwand, Ausbildung eines hypoplastischen Beckens und/oder Subluxation des Hüftkopfs oder Ausbildung einer Coxa magna oder anderer Wachstumsstörungen am proximalen Femur kommen.

Therapeutisch kommen Pfannendachplastiken, Beckenosteotomien und/oder varisierende intertrochantäre Osteotomien infrage.

Um diese Komplikationen rechtzeitig zu erkennen, sind jährliche klinisch-radiologische Kontrollen bis zum Wachstumsabschluss zu fordern.

\section{Langzeitergebnisse}

Die Spätergebnisse nach kindlichen Azetabulumfrakturen wurden am genausten von Heeg untersucht [7]. Er fand bei 6 Patienten eine Beteiligung der Wachstumsfuge. Nach durchschnittlich 8 Jahren wurden die Kinder nachuntersucht. Nach radiologisch diagnostizierten Salter-Harris-Typ-I- + -II-Frakturen traten keine Wachstumsstörungen auf, das funktionelle Ergebnis war sehr gut. Bei den 2 Typ-V-Verletzungen kam es zum vorzeitigen Fugenverschluss, teilweise mit Subluxation des Hüftkopfs. Bei bei- den Kindern erfolgte eine Korrekturoperation. In einer weiteren Analyse von 23 kindlichen Azetabulumfrakturen bis zum 17. Lebensjahr wurde das radiologische und funktionelle Ergebnis mittels Harris-Score beurteilt. Nach konservativer Behandlung waren die Resultate akzeptabel. Salter-Harris Typ-V-Verletzungen zeigten schlechtere Ergebnisse. Die besten Ergebnisse wiesen Patienten mit weniger als $2 \mathrm{~mm}$ Dislokation auf. Allgemein waren die funktionellen Ergebnisse besser als die radiologischen.

In einer Multicenterstudie analysierten Heeg et al. 29 Patienten bis zum 16. Lebensjahr mit Azetabulumfraktur [5]. Zehn dieser Patienten müssen als Adoleszenten angesehen werden (15-16 Jahre alt) und wurden überwiegend $(n=7)$ osteosynthetisch versorgt. Von den übrigen 19 Patienten mit kindlichen Azetabulumfrakturen wurden nur 5 operativ stabilisiert, von denen 2 Langzeitprobleme durch Ausbildung einer Hüftkopfnekrose sowie einer Ankylose aufwiesen. In der Gruppe der konservativ behandelten Kinder traten keine signifikanten Langzeitfolgen auf.

\section{Literatur}

${ }^{1}$ Alpar EK, Owen $R$. Injuries of the Pelvis and Hip. In: Alpar EK, Owen R, eds. Paediatric Trauma. Tunbridge Wells, Kent: Castle House Publications Ltd; 1988

2 Bucholz R, Ezaki M, Ogden J. Injury of the acetabular triradiate physeal cartilage. J Bone Joint Surg [Am] 1982; 64: 600-609

3 Currey J, Butler G. The mechanical properties of bone tissue in children. J Bone Joint Surg [Am] 1975; 57: 810-814

${ }^{4}$ Grisoni N, Connor S, Marsh E et al. Pelvic fractures in a pediatric level I trauma center. J Orthop Trauma 2002; 16: 458-463

${ }^{5}$ Heeg M, de Ridder VA, Tornetta 3rd Pet al. Acetabular fractures in children and adolescents. Clin Orthop Relat Res 2000; 376: 80-86
${ }^{6}$ Heeg M, Klasen H, Visser J. Operative treatment for acetabular fractures. J Bone Joint Surg [Br] 1990; 72: 383-386

7 Heeg M, Visser J. Acetabular fractures involving the growth plate. Acta Ortop Scand 1988; 59: 755

${ }^{8}$ Meyer-Junghänel L, Gänsslen A, Pohlemann T et al. Behandlungsergebnisse nach komplexem Beckentrauma bei Kindern. Unfallchirurg 1997: 100: 225-233

9 Pohlemann T, Gänsslen A, Partenheimer A. Kapitel 18: Becken und Acetabulumverletzungen im Kindesalter. In: Weinberg AM, Tscherne H, eds. Unfallchirurgie im Kindesalter. Heidelberg: Spinger; 2006: 577-602

10 Ponseti I. Growth and development of the acetabulum in the normal child. J Bone Joint Surg [Am] 1978; 60: 575-585

11 Schlickewei W, Keck T. Pelvic and acetabular fractures in childhood. Injury 2005; 36: S-A57-S-A63

12 Silber J, Flynn J. Changing patterns of pediatric pelvic fractures with skeletal maturation: implications for classification and management. J Ped Orthop 2002; 22: 22-26

13 Trousdale R, Ganz R. Posttraumatic acetabular dysplasia. Clin Orthop Relat Res 1994; 305: 124-132

\section{Dr. med. Axel Gänsslen}

Oberarzt

Klinik für Unfallchirurgie, Orthopädie und Neurotraumatologie Allgemeines Krankenhaus Celle Siemensplatz 4 29223 Celle

dr.gaensslen@gmx.de

Associate Professor PHD med. Annelie-Martina Weinberg Oberärztin

LKH Universitätsklinik für Kinder- und Jugendchirurgie Auenbruggerplatz 34

8036 Graz

Österreich

annelie.weinberg@t-online.de 